\title{
Drawing materials studied by THz spectroscopy
}

\section{Andrea Taschin ${ }^{1,2}$, Paolo Bartolini ${ }^{1}$, Jordanka Tasseva ${ }^{3}$, Jana Striova ${ }^{4}$, Raffaella Fontana ${ }^{4}$, Cristiano Riminesi ${ }^{5}$, Renato Torre ${ }^{1,2}$}

${ }^{1}$ European Lab. for Non-Linear Spectroscopy (LENS), Univ. di Firenze, via N. Carrara 1,I-50019 Sesto Fiorentino, Firenze, Italy.

${ }^{2}$ Dip. di Fisica e Astronomia, Univ. di Firenze, via Sansone 1, I-50019 Sesto Fiorentino, Firenze, Italy.

${ }^{3}$ INFN, Istituto Nazionale di Fisica Nucleare, Sez. di Napoli, Complesso Univ. di M. S. Angelo, Ed. 6- Via Cintia, 80126 Napoli.

${ }^{4}$ Istituto Nazionale di Ottica, INO-CNR, Largo Fermi 6, I-50125 Firenze, Italy.

${ }^{5}$ Istituto per la Conservazione e la Valorizzazione dei Beni Culturali, ICVBC-CNR, Via Madonna del Piano 10, I-50019 S Sesto Fiorentino, Italy.

\section{ABSTRACT}

THz time-domain spectroscopy in transmission mode was applied to study dry and wet drawing inks. In specific, cochineal-, indigo- and iron-gall based inks have been investigated; some prepared following ancient recipes and others by using synthetic materials. The THz investigations have been realized on both pellet samples, made by dried inks blended with polyethylene powder, and layered inks, made by liquid deposition on polyethylene pellicles. We implemented an improved THz spectroscopic technique that enabled the measurement of the material optical parameters and thicknesses of the layered ink samples on absolute scale. This experimental investigation shows that the THz techniques have the potentiality to recognize drawing inks by their spectroscopic features.

\section{Section: RESEARCH PAPER}

Keywords: ink; drawing material; THz spectroscopy

Citation: Andrea Taschin, Paolo Bartolini, Jordanka Tasseva, Jana Striova, Raffaella Fontana, Cristiano Riminesi, Renato Torre, Drawing materials studied by THz spectroscopy, Acta IMEKO, vol. 6, no. 3, article 3, September 2017, identifier: IMEKO-ACTA-06 (2017)-03-03

Editor: Sabrina Grassini, Politecnico di Torino, Italy

Received February 13, 2017; In final form July 27, 2017; Published September 2017

Copyright: (C) 2017 IMEKO. This is an open-access article distributed under the terms of the Creative Commons Attribution 3.0 License, which permits unrestricted use, distribution, and reproduction in any medium, provided the original author and source are credited

Funding: Regione Toscana, POR-CROFSE-UNIFI-26 ; Ente Cassa di Risparmio Firenze, N. 2015-0857 and IPERION CH - GA 654028

Corresponding author: R. Torre, e-mail: torre@lens.unifi.it

\section{INTRODUCTION}

During the last years, $\mathrm{THz}$ spectroscopy has been intensely developed thanks to the realization of new laser sources capable of generating coherent radiation. Because the $\mathrm{THz}$ radiation is of low energy, $\mathrm{THz}$ spectroscopic techniques are nondestructive tools able to provide valuable information in the cultural heritage field. Recently, some studies [1]-[3] have demonstrated the possibility to use this radiation in the investigation of painting and drawing media. In particular, the THz-Time Domain Spectroscopy (THz-TDS) proved to be a powerful $\mathrm{THz}$ spectroscopic technique able to measure different optical parameters of the artistic works. These pulsed $\mathrm{THz}$ techniques can be implemented by using both the transmission or reflection configurations [4]-[8].

When a $\mathrm{THz}$ pulse passes through the sample, the material induces modifications in its temporal waveform; from the analysis of these variations the optical parameters of the material can be recovered. Nevertheless, the extraction of the transmission parameters (i.e. absorption coefficients and indexes of refraction) from the $\mathrm{THz}$ pulse waveform is not a trivial task [9]. It depends on the experimental conditions and sample characteristics; in particular, the procedure becomes very complex for thin film samples [10].

Recently, we have presented a $\mathrm{THz}$ spectroscopic study of layered ink samples [7], [8]. We implemented an innovative experimental and analytical method enabling a reliable extraction of the $\mathrm{THz}$ parameters from a TDS experiment. We report here on the investigation of a series of black and colored inks by means of this innovative THz-TDS approach. We measured both bulk inks in pellet samples and inks layered as thin film samples. Moreover, we compared the ink $\mathrm{THz}$ spectra with those of the separate specific constituents of the ink pigments. This enables one to identify the origin of the spectroscopic peaks present in the $\mathrm{THz}$ absorption spectra of the inks. 


\section{MATERIALS AND METHODS}

\subsection{Samples preparation}

We investigated samples prepared in the laboratory using home-made and commercial inks. The former inks have been prepared using both ancient recipes (Giovanni Alcherio 1411) and synthetic materials. Four groups of inks, according to the colour they render, might be distinguished: red, blue, black and white. In Table 1, we report the compositions of the studied inks and the recipes followed for their preparations. The black inks, all iron gall based, were prepared in the laboratory following two main recipes containing either oak galls as a source of the gallo-tannic acid (Recipe A in Table 1) or preliminary synthesized gallic acid (Recipe B in Table 1). Details about the ink preparation are reported in [7].

Dried inks were blended with polyethylene (PE) powder (Merck), ground, and pressed in pellets of $13.2 \mathrm{~mm}$ diameter and thickness around $1 \mathrm{~mm}$. PE is the ideal support for absorption spectroscopy in the $\mathrm{THz}$ region thanks to its negligible absorption coefficient (below $1 \mathrm{~cm}^{-1}$ see [11] and references therein). The analyte concentration in the PE pellet was chosen to be approximately $33 \mathrm{wt}$. \% that, for most of the studied inks, is an optimal concentration, rendering detectable any eventual features in a relatively wide spectral range. As an attempt of getting closer to a real-practice experiment on ancient manuscripts and drawings, some of the above inks have been studied also in the form of thin films, of the order of tens of $\mu \mathrm{m}$, layered on $10 \mu \mathrm{m}$ PE pellicles (polyethylene far-IR sample cards by Sigma-Aldrich).

\subsection{Terahertz time domain spectroscopy}

A standard THz-TDS set-up in transmission configuration has been used to extract the values of the refractive index, $n$, and the absorption coefficient, $\alpha$, of inks in the frequency range $0.1-4 \mathrm{THz}$. THz pulses are produced by exciting a biased photoconductive antenna with femtosecond optical laser pulses $(\lambda=780 \mathrm{~nm}, \Delta t=120 \mathrm{fs}$ at $100 \mathrm{MHz})$. The outgoing $\mathrm{THz}$ radiation is guided, as usual, by two couples of off-axis parabolic mirrors. The THz field is collimated, focused on the sample, collimated again, and finally focused on a second photoconductive antenna for its detection. A second optical laser pulse, sent to the detection antenna, acts as the current gate for the $\mathrm{THz}$ detection. The temporal evolution of the photocurrent amplitude in the detection antenna, acquired by changing time delay between exciting and gate pulses, is directly related to the electric field amplitude of the $\mathrm{THz}$ radiation. This current is amplified by a lock-in amplifier and digitized by an acquisition board. The processed signal and delay line encoding are acquired by homemade software for retracing the final time dependent $\mathrm{THz}$ field. The whole $\mathrm{THz}$ set-up is enclosed in a nitrogen purged chamber for removing the water vapour contribution present at the $\mathrm{THz}$ frequencies spanned by the experiment.

More details about the used THz-TDS set-up can be found in [7], [8] and the supplementary information therewith.

\subsection{Materials optical parameters extraction}

The transfer function of the material, $H(\omega)$ [7], [8] is the ratio between the $\mathrm{THz}$ field transmitted after the sample, $E_{\mathrm{t}}(\omega)$, and the incident field, $E_{\mathrm{i}}(\omega)$. It expresses how the phase and amplitude of an electromagnetic plane wave of frequency $\omega$ change due to the absorption and refraction of the crossed medium. For a homogeneous dielectric slab of thickness $d$ and complex refractive index $\hat{n}$, surrounded by nitrogen, and for normal incidence, the theoretical expression of the transfer function can be written as

$$
\begin{aligned}
& H(\omega)=\frac{E_{t}(\omega)}{E_{i}(\omega)}=\tau \tau^{\prime} \exp \left\{-i\left[\hat{n}(\omega)-n_{0}\right] \frac{\omega d}{c}\right\} \cdot F P(\omega) \\
& \text { with } F P(\omega)=\sum_{m=0}^{\infty}\left\{\rho^{\prime 2} \exp \left[-2 i \hat{n}(\omega) \frac{\omega d}{c}\right]\right\}^{m}
\end{aligned}
$$

being: $\tau=2 /\left(n_{0}+\hat{n}\right)$ and $\tau^{\prime}=2 \hat{n} /\left(n_{0}+\hat{n}\right)$ the nitrogensample and sample-nitrogen complex transmission coefficients, respectively; $\quad \rho^{\prime}=\left(n_{0}-\hat{n}\right) /\left(n_{0}+\hat{n}\right) \quad$ the reflection coefficients, with $\hat{n}=n(\omega)-i k(\omega)$, where $n(\omega)$ is the refractive index, $k(\omega)$ the extinction coefficient, and $n_{0}$ the refractive index of nitrogen, $c$ is the vacuum speed of light. The multiple reflections inside the sample are taken into account by the Fabry-Pérot effect defined by the $F P(\omega)$ function.

The optical properties of the material, $n(\omega)$ and $\alpha(\omega)=$ $\frac{2 \omega}{c} k(\omega)$ and its thickness, $d$, in principle, can be completely characterized by measuring the experimental transfer function, $H_{\text {exp }}(\omega)$, obtained by the ratio of the complex Fourier transform of the sample and the reference signal. Unfortunately, the expression for $H(\omega)$ is not expressed in a closed-form and the real thickness of the sample is not generally known with enough accuracy. Some numerical iterative process has to be used to extract $n(\omega), \alpha(\omega)$ and $d$ [12]-[16] and this process depends on the nature of the $\mathrm{THz}$ signal and the thickness of samples.

Figure 1 reports typical signals measured by our $\mathrm{THz}$-TDS set-up on Black B pellet sample. Both the temporal evolution of the THz signal (main panel of Figure 1, oscillations in the 5-10 ps range of the red curve) and its spectral content (inset of Figure 1, holes in the spectrum) show modifications due to the

\begin{tabular}{|c|c|c|}
\hline Color Ink & Ink type & Ink Recipes \\
\hline \multirow[t]{2}{*}{ Red } & 1 & Commercial cochineal carmine: possibly based on carminic acid $\left(\mathrm{C}_{22} \mathrm{H}_{20} \mathrm{O}_{13}\right)$. \\
\hline & 2 & Red ochre: containing iron(III)oxide-hydroxide. \\
\hline Blue & & Commercial blue ink: based on 2,2-Bis(2,3-dihydro-3-oxoindolyli-den), Indigotin. \\
\hline \multirow[t]{3}{*}{ Black } & A & $\begin{array}{l}\text { Recipe A - Iron gall ink with Arabic gum: } 70 \mathrm{~mL} \text { water, } 10 \mathrm{~mL} \text { white wine, } 10 \mathrm{~mL} \text { red vinegar, } 5 \mathrm{~g} \text { powdered } \\
\text { oak galls (Bizzarri, Firenze), } 1.25 \mathrm{~g} \text { Arabic gum, } 1.25 \mathrm{~g} \mathrm{FeSO}_{4} \cdot 7 \mathrm{H} 2 \mathrm{O} \text { (Bizzarri, Firenze) melanterite. }\end{array}$ \\
\hline & B & $\begin{array}{l}\text { Recipe B - Iron gall ink with Arabic gum: } 14 \mathrm{~mL} \text { distilled water, } 1.14 \mathrm{~g} \text { gallic acid (Bizzarri, Firenze), } 0.29 \mathrm{~g} \\
\text { Arabic gum, } 0.29 \mathrm{~g} \mathrm{FeSO}_{4} \cdot 7 \mathrm{H} 2 \mathrm{O} \text { (Bizzarri, Firenze). }\end{array}$ \\
\hline & $\mathrm{C}$ & Commercial iron gall ink (Zecchi). \\
\hline White & & White lead: $2 \mathrm{PbCO}_{3} \cdot \mathrm{Pb}(\mathrm{OH})_{2}$. \\
\hline
\end{tabular}

Table 1. Compositions and recipes of the studied inks. 


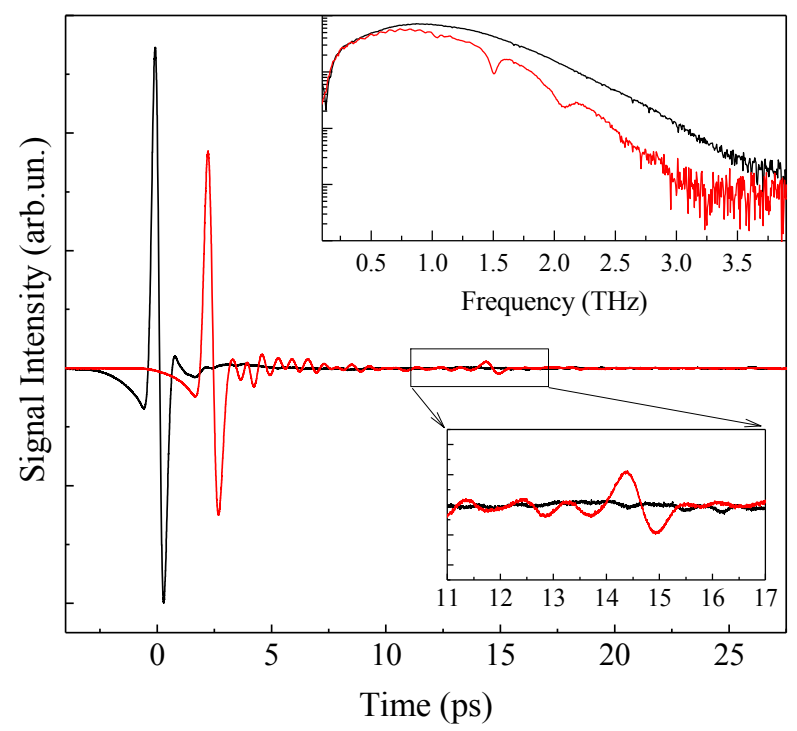

Figure 1. Main panel: typical time evolution of the $\mathrm{THz}$ electric field of the reference (black line) and signal (red line) in the Black B pellet sample. Inset: amplitude spectra of the signals obtained by their Fourier transforms. In the signal data, the oscillations after the main pulse are due to the ink absorption effects whilst the little pulse at 14.5 ps to the FP effect, see enlargement.

absorption of the ink. In time evolution of the sample $\mathrm{THz}$ signal (red curve), the additional little pulse at around 15 after the main pulse is due to the internal reflections of the $\mathrm{THz}$ pulse between the sample surfaces. These appear in the spectrum as fast oscillations in the low frequency window.

If the reflections are clearly distinguishable in the time domain and they are not superimposed on the oscillating modifications due to the sample absorption (contrary to what is observed in Figure 1), the analysis for extraction of the optical parameters is relatively simple [7], [8]. Usually, this occurs with thick samples and weak absorption.

Contrarily, in a thin-layered sample, the reflection signals are close in time and partially superimposed, so a correct extraction of the material parameters requires a complex data analysis where the multi-reflection processes are properly taken into account [7]-[9]. The transfer function becomes more complex and the material parameters, $n(\omega)$ and $\alpha(\omega)$, can be extracted only using an articulated data analysis based on iterative fitting procedures [7]-[10]. Otherwise, the refractive index and absorption coefficient spectra are distorted by fake oscillations.

Recently, we have implemented an innovative experimental procedure and numerical method to analyse the transmission THz-TDS signal of samples composed of multiple thin layers [7], [8].

The numerical method is based on a polynomial fit of the optical parameters, by which we can catch the real physical frequency behaviour and remove the FP oscillations. We perform a preliminary evaluation of $n(\omega), k(\omega)$ and $d$ : neglecting the FP term and the imaginary part of the refractive index in the Fresnel coefficients of (1), an analytical expressions for $n(\omega)$ and $k(\omega)$ can be obtained as a function of $H(\omega)$ with $d$ measured by a micrometer screw. Then we perform a polynomial fit of $n(\omega)$ and $k(\omega)$ in order to remove the FP oscillations by which they are affected. Then we minimize the difference between the calculated and experimental $H$ functions integrated over all the frequencies, $\Delta H$, to infer new best values for $n(\omega), k(\omega)$ and $d$. The final step concerns the refinement of the frequency dependence of parameters $n(\omega)$ and $k(\omega)$ obtained performing a minimization of the transfer function difference, $\Delta H(\omega)$, at variable frequencies and keeping fixed the $d$ value.

This data analysis enables a reliable and robust extraction of the frequency dependence of the optical parameters, opening the possibility to measure even weak and broad peaks present in the $n(\omega)$ and $k(\omega)$ functions. Moreover, it allows measuring the sample layer dimensions, even when it is not sufficiently thick to generate visible reflections in the time-domain data.

In this experimental investigation, we applied this method on the THz-TDS data from a double layered sample: an ink film deposited on polyethylene (PE) pellicles.

All details about the data analysis on single and dual thin layers are reported in [7] and the supplementary information enclosed therewith and in [8].

\section{RESULTS AND DISCUSSION}

Due to the complexity in calculating the molar concentration of inks in pellets, only the relative molar absorption coefficient and the refractive index may be extracted and these are reported in relative units (Figures 2-6). For the pellet samples, scattering processes due to inhomogeneities in the sample could also affect the optical properties. In particular, the mismatch between the ink and PE indices gives rise to a spectral distortion called Christiansen Effect [17]. The effect can be greatly reduced by a fine grinding of the powders. Usually, the scattering introduces an additional absorption contribution, which grows with frequency, and distorts the absorption peaks and their dispersion curves. Yet, new fake absorption peaks could arise, as a result of interference of Mie scattering that, however, do not exhibit the characteristic dispersion in the refractive index. As long as the peaks in the measured

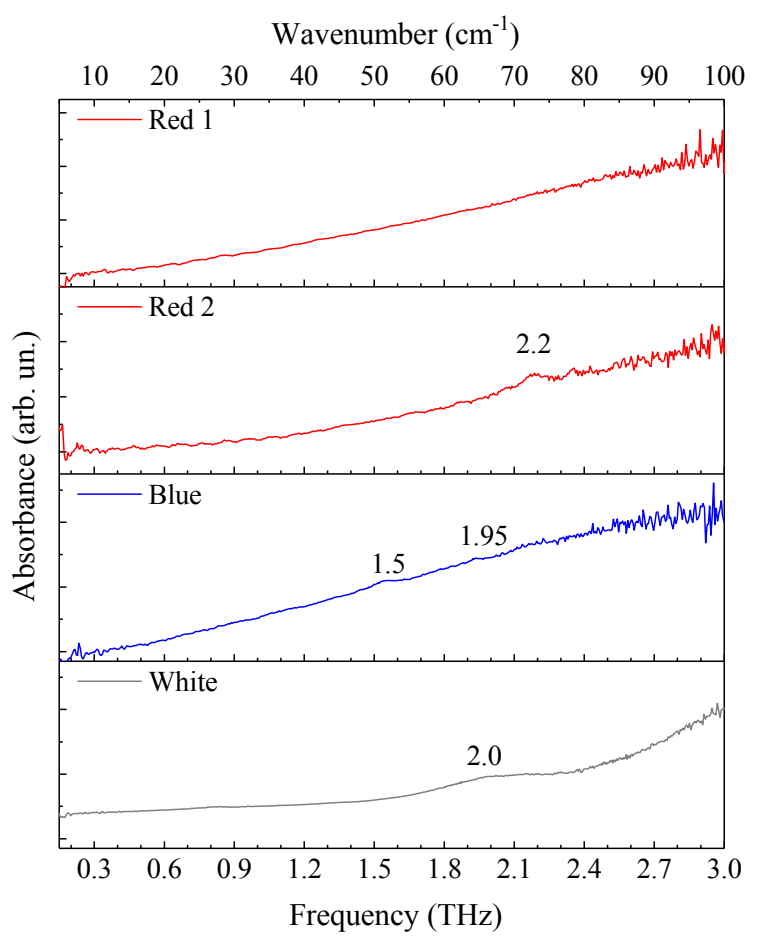

Figure 2. THz absorbance spectra for Red 1, Red 2, Blue, and White pigments measured on pellets. Some weak spectral contributions are evident in all the inks except in the cochineal carmine ink. 


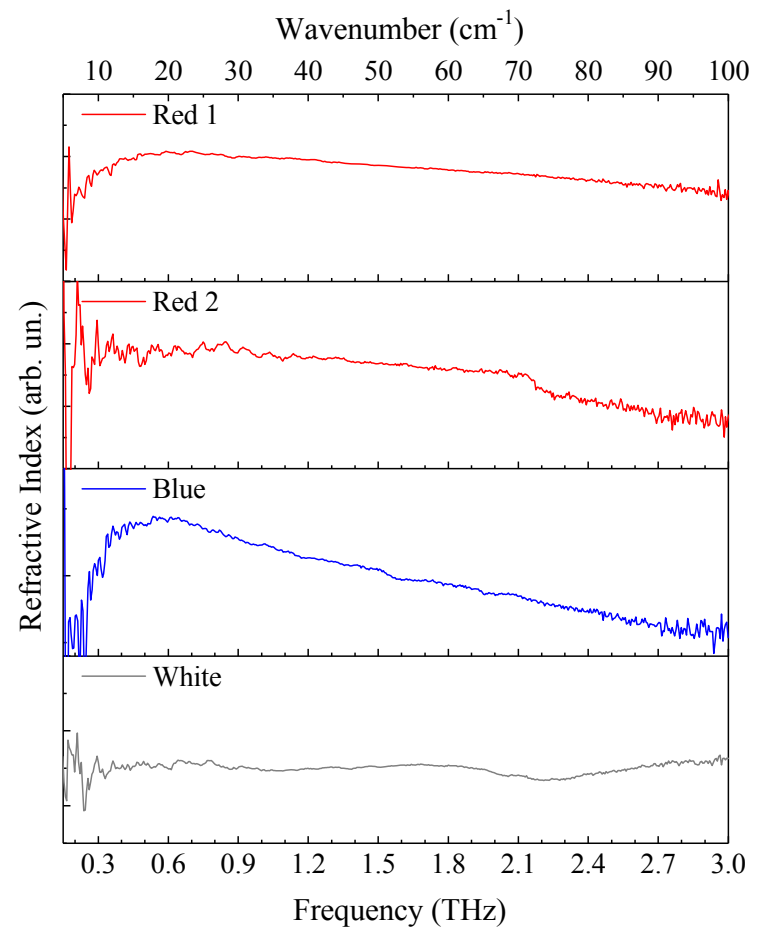

Figure 3. Frequency dependence of the refractive indices for Red 1, Red 2, Blue, and White pigments measured on pellets. The dispersion behaviour confirms the absorptive origin of the features found in the absorbance spectra of the Red 2, Blue, and White samples in the 1.5-2.5 THz range.

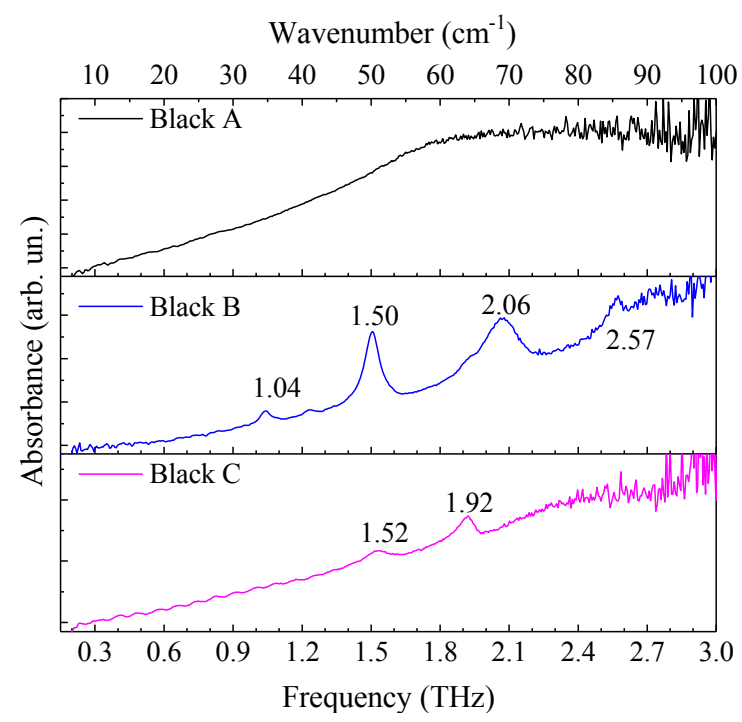

Figure 4. THz absorbance spectra for the iron gall inks measured on pellets. The pigments prepared following the ancient recipe (Recipe A) containing oak galls (Black A) do not show any particular spectral feature contrary to what is observed in the inks containing synthesized gallic acid (Black B and Black C) which instead shows well pronounced peaks.

absorbance spectra are of a symmetric Lorentzian line shape and are also present in the relative dispersions of the refractive index, the scattering processes can be considered negligible.

For the films, instead, the employed analysis allows the measurement of the optical properties of the same ink layer, and the absolute absorption coefficients and refractive indices are reported (Figure 7).

In Figure 2 we report the absorbance spectra of Red 1, Red 2, Blue, and White inks in pellets. Only Red 1 shows featureless

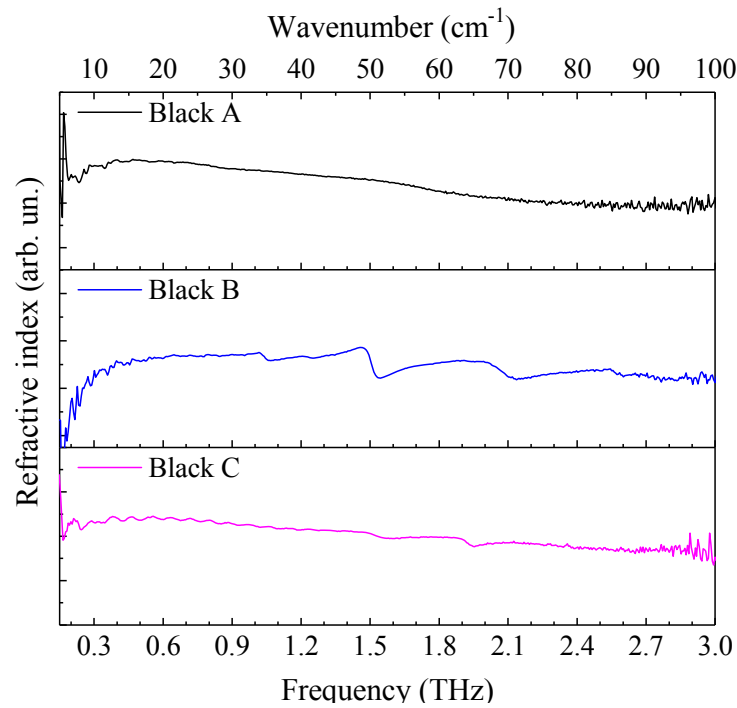

Figure 5. Frequency behaviour of the refractive indices for the iron gall inks measured on pellets. The dispersive features of the refractive index for the Black $B$ and Black $C$ inks confirm what measured in the respective absorbance spectra.

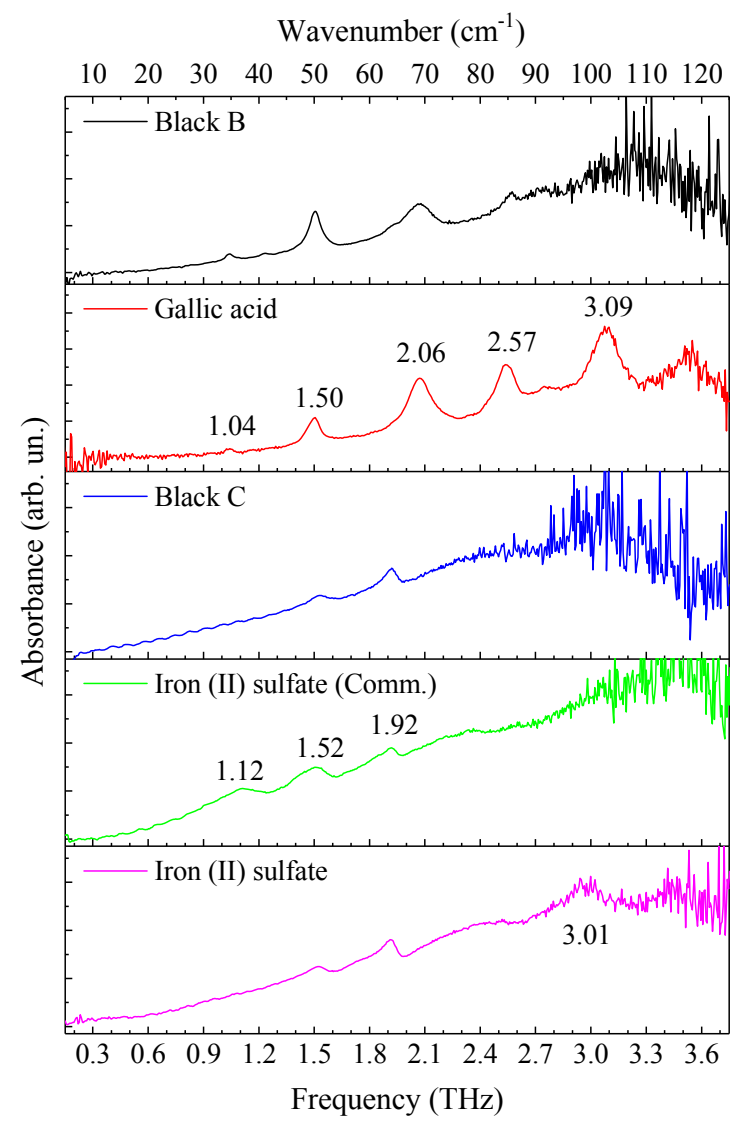

Figure 6. $\mathrm{THz}$ absorbance spectra for the Black B and Black $\mathrm{C}$ pigments together with those of the individual constituents gallic acid, iron(II) sulfate commercial, and iron(II) sulfate synthesized in the laboratory.

spectra in the measured $\mathrm{THz}$ range whilst the others present a few spectral peaks. In particular, Red 2 shows a peak around 2.2 $\mathrm{THz}$, Blue two very weak peaks at 1.5 and $1.95 \mathrm{THz}$ and the White ink a broad band centred at $2 \mathrm{THz}$. These features are confirmed by the dispersion sample visible in Figure 3. 

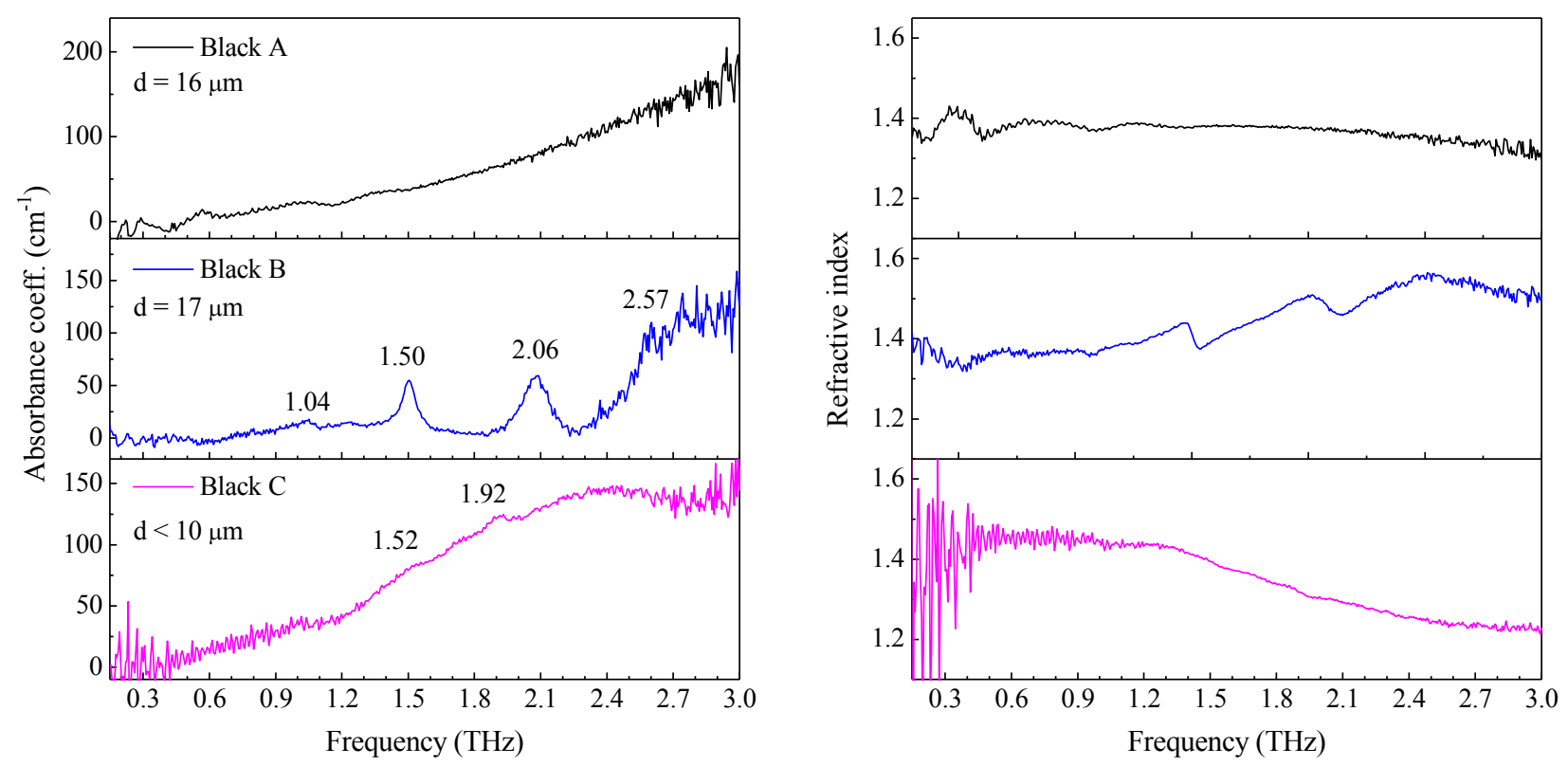

Figure 7. Absorption coefficient (left panel) and refractive index (right panel) as a function of frequency for the black inks on PE pellicles.

Figures 4 and 5 show the absorbance spectra and frequency dependence of the refractive indexes for all three black inks measured on pellets. The iron gall inks prepared following the old recipe of mixing mainly iron(II) sulfate and oak galls powder, Black $\mathrm{A}$, shows a featureless response in the $\mathrm{THz}$ range. On the other hand, the ink containing synthetic gallic acid, Black B, and the commercial iron gall ink, Black C, show structured spectra with several peaks. Our results are in perfect agreement with those reported by Bardon et al. [6].

In order to ascribe each spectral component of Black B and Black C, we compared them with those of the individual constituents, gallic acid, iron(II) sulfate commercial, and iron(II) sulfate synthesized in the laboratory (Figure 6). Gallic acid shows well-defined bands around 1.04, 1.50, 2.06, 2.57 and 3.09 $\mathrm{THz}$. In the spectra of commercial ferrous sulfate, features at $1.12,1.52,1.92 \mathrm{THz}$ are present. For the in-laboratory synthesized ferrous sulfate, instead, the peak at $1.12 \mathrm{THz}$ disappears and an additional band around 3.01 THz is detected. The iron gall inks prepared following the recipe A do not show any features related either to gallic acid or to iron(II) sulfate. It is known that under hydrolysis gallic acid is extracted from the oak galls. Most likely all ferrous sulfate has oxidized to ferric sulfate, resulting in the formation of iron-tannic complexes. That hypothesis is well supported by Bardon et al. [6], reporting no contribution of tannic acid in the $0.15-3 \mathrm{THz}$ range and a single, questionable feature in the spectrum of iron(III) sulfate.

On the other hand, if gallic acid was extracted from the oak galls, and the iron sulfate to gallic acid molar ratio was close to 1 , the two components might have been consumed in the formation of iron gallate complexes, possibly featureless in the studied range. In the spectra of the laboratory-made iron gall ink prepared from ferrous sulfate and synthesized gallic acid (recipe B, Table 1), we can see the response of the gallic acid, while no ferrous sulfate contribution is present (Figure 6). Following what was reported by other researchers [6], we could attribute this to the low iron sulfate to gallic acid molar ratio that in our case is 0.1 . The commercially available iron gall ink, instead, shows features of the iron sulfate and lacks those of gallic acid as reported in Figure 6.
As a next step we decreased the analyte quantity, preparing thin films on transparent PE pellicles. The analysed inks were: the three black inks, Black A, Black B, and Black C and their optical parameters are shown in Figure 7 . We were able to obtain also in this case a very good signal-to-noise ratio and to get agreement with the results from bulk samples. The bilayer analysis procedure [7], [8] enables us to measure thicknesses down to $10 \mu \mathrm{m}$. Iron-gall ink, prepared with oak galls powder, once again does not manifest features of either gallic acid or ferrous sulfate. Similarly to the pellet sample, the layered irongall ink prepared following recipe $\mathrm{B}$ reveals the presence of gallic acid by peaks in absorbance at 1.04, 1.50, 2.06 and 2.57 THz. The film from commercial iron-gall ink shows the 1.92 $\mathrm{THz}$ feature of ferrous sulfate, while the one at $1.52 \mathrm{THz}$ seems to be strongly damped.

It is worth pointing out that, thanks to the study on the thin films on PE pellicles, the refractive index and absorption coefficient values that we measured are absolute values different to what was measured in the pellet samples.

\section{CONCLUSIONS}

$\mathrm{THz}$ time-domain spectroscopy in transmission configuration was applied to investigate inks commonly used in artworks, i.e. red, indigotin-based, iron-gall and lead white inks. Pellet samples were prepared, blending the bulk dried pigments with PE powder. Thin film samples were made by liquid inks layered on PE pellicles and subsequently dried. The THz-TDS technique achieved high signal-to-noise ratio for both pellet and layered inks, enabling a particularly meaningful analysis of the data. The $\mathrm{THz}$ spectra measured show similar features in both sample types, pellet and film.

In particular, the red cochineal carmine does not show any evident spectral signature in the 0.1-3.2 THz range, whereas the red ochre shows a weak but distinct absorption peak at about 2.2 THz. The blue ink presents two weak absorption peaks at 1.5 and $1.95 \mathrm{THz}$. The absorbance spectrum of white lead shows a shallow feature at around $2 \mathrm{THz}$. 
The spectroscopic features of the iron gall inks are more articulated and pronounced; we want to stress how the inks prepared by the ancient recipe (recipe A), can be immediately distinguished from those prepared with synthetic materials (recipe B) or commercial ones. In fact, the black inks prepared by recipe A show a featureless response. On the contrary, black inks prepared by recipe B or commercially available inks reveal the spectral signatures of their elementary constituents.

Moreover, the possibility to measure in absolute scale the $\mathrm{THz}$ spectra of layered inks brings these spectroscopic investigations closer to real-practice problems. In these respects, the present research is a rare attempt to improve the THz-TDS method in order to overcome the gap between the purely scientific uses and the operative applications in the field of cultural heritage.

\section{ACKNOWLEDGEMENT}

This work was funded by Regione Toscana, prog. PORCROFSE-UNIFI-26 and by Ente Cassa di Risparmio Firenze, prog. 2015-0857. The authors of INO-CNR acknowledge IPERION CH project GA 654028, funded by the EU community's H2020-research infrastructure program.

\section{REFERENCES}

[1] K. Fukunaga, Y. Ogawa, S.i. Hayashi, I. Hosako, Application of terahertz spectroscopy for character recognition in a medieval manuscript, IEICE Electronics Express, 5, (2008), pp. 223-228.

[2] C. Seco-Martorell, V. Lopez-Dominguez, G. Arauz-Garofalo, A. Redo-Sanchez, J. Palacios, J. Tejada, Goya's artwork imaging with Terahertz waves, Opt Express, 21, (2013), pp. 17800-17805.

[3] G.C. Walker, J.W. Bowen, W. Matthews, S. Roychowdhury, J. Labaune, G. Mourou, M. Menu, I. Hodder, J.B. Jackson, Subsurface terahertz imaging through uneven surfaces: visualizing Neolithic wall paintings in Catalhoyuk, Opt Express, 21, (2013), pp. 8126-8134.

[4] K. Fukunaga, M. Picollo, Terahertz spectroscopy applied to the analysis of artists' materials, Appl Phys a-Mater, 100, (2010), pp. 591-597.

[5] I. Cacciari, J. Agresti, S. Siano, Combined THz and LIPS analysis of corroded archaeological bronzes, Microchemical Journal, 126 (2016), pp. 76-82.
[6] T. Bardon, R.K. May, P.F. Taday, M. Strlic, Systematic study of terahertz time-domain spectra of historically informed black inks, Analyst, 138, (2013), pp. 4859-4869.

[7] J. Tasseva, A. Taschin, P. Bartolini, J. Striova, R. Fontana, R. Torre, Thin layered drawing media probed by $\mathrm{THz}$ time-domain spectroscopy, Analyst, 142, (2017), pp. 42-47.

[8] A. Taschin, J. Tasseva, P. Bartolini, R. Torre, THz time-domain spectroscopic investigations of thin films, Measurement (2017), http://dx.doi.org/ 10.1016/j.measurement.2017.05.074.

[9] W. Withayachumnankul, M. Naftaly, Fundamentals of Measurement in Terahertz Time-Domain Spectroscopy, Journal of Infrared, Millimeter, and Terahertz Waves, 35, (2013), pp. 610-637.

[10] J.F. O'Hara, W. Withayachumnankul, I. Al-Naib, A Review on Thin-film Sensing with Terahertz Waves, Journal of Infrared, Millimeter, and Terahertz Waves, 33, (2012) pp. 245-291.

[11] Y.S. Lee, Principles of terahertz science and technology, Springer US, New York, (2009), ISBN 978-0-387-09540-0.

[12] W. Withayachumnankul, B .Ferguson, T. Rainsford, S. P. Mickan, D. Abbott, Material parameter extraction for terahertz time-domain spectroscopy using fixed-point iteration Proc. SPIE, 5840, (2005), pp. 221-231.

[13] I. Pupeza, R. Wilk, M. Koch, Highly accurate optical material parameter determination with $\mathrm{THz}$ time-domain spectroscopy, Opt. Express, 15, (2007), pp. 4335-4350.

[14] M. Scheller, Real-time terahertz material characterization by numerical three-dimensional optimization, Opt. Express, 19, (2011), pp. 10647-10655.

[15] M. Scheller, C. Jansen, M. Koch, Analyzing sub-100- m samples with transmission terahertz time domain spectroscopy, Opt. Commun., 282, (2009), pp. $1304-1306$.

[16] M. Scheller, M. Koch, Fast and Accurate Thickness Determination of Unknown Materials using Terahertz Time Domain Spectroscopy, J. Infrared Millim. Terahertz Waves, 30, (2009), pp. 762-769.

[17] M. Franz, B.M. Fischer, M. Walther, The Christiansen effect in terahertz time-domain spectra of coarse-grained powders, App. Phys. Lett., 92, (2008) pp. 021107. 